Take the online multiple choice questions associated with this article (see page 1158)

Heart 2007; 93:1137-1146. doi: 10.1136/hrt.2003.025270

- he aim of this paper is to review the clinical epidemiology of heart failure. The last paper comprehensively addressing the epidemiology of heart failure in Heart appeared in $2000 .^{\mathrm{wl}}$ Despite an increase in manuscripts describing epidemiological aspects of heart failure since the 1990s, ${ }^{1}$ additional information is still needed, as indicated by various editorials. ${ }^{\text {w2 }}$ w3

The evaluation and management of heart failure is schematically depicted in fig l. Following some methodological considerations, most issues indicated in fig 1 (risk factors, aetiology, prevalence, incidence, prognosis, prevention) will be discussed.

The therapeutic management of patients diagnosed with heart failure is beyond the scope of this paper, as is detailed information about the possible diagnostic tests and strategies to establish or rule out heart failure, although the prevailing definitions and categorisations of the syndrome will be discussed. The guidelines of the European Society of Cardiology and selected reviews provide up to date information on the diagnosis and therapeutic management of heart failure. ${ }^{2}$ w4 w5

\title{
DEFINITION AND CLASSIFICATION OF HEART FAILURE
}

Heart failure is a syndrome with symptoms and signs caused by cardiac dysfunction, resulting in reduced longevity. To establish a diagnosis of heart failure, the European Society of Cardiology guidelines warrant the presence of symptoms and signs (tables 1 and 2), objective evidence of cardiac dysfunction (preferably by echocardiography), and, in case of remaining doubt, a favourable response to treatment directed towards heart failure. ${ }^{\mathrm{w} 5}$ To support the failing heart numerous compensatory mechanisms occur, including activation of the neurohormonal system. ${ }^{2}$ An increase in natriuretic peptide concentrations (particularly B type natriuretic peptide) is considered a hallmark of heart failure.

The diagnosis of heart failure, especially when relying solely on symptoms and signs (which is often the case in primary care), is fraught with difficulties. ${ }^{3}$ Many patients deemed to have heart failure will simply be found to be obese, have a poor physical condition, pulmonary disease, or ischaemia on further examination. Evidence is accumulating that normal natriuretic peptide levels and a normal electrocardiogram should lead to a reconsideration of a diagnosis of heart failure. ${ }^{4 \text { w6 }}$

\section{Acute versus chronic heart failure}

Heart failure generally is a chronic condition (chronic heart failure-CHF) in which bouts of worsening symptoms and signs can occur that may require hospitalisation or more frequent doctor visits (decompensation of CHF). Alternatively, heart failure may present acutely, with occurrence of severe symptoms and signs within $24 \mathrm{~h}$. Acute heart failure clinically presents in several forms:

- acute pulmonary oedema secondary to cardiac dysfunction

- cardiogenic shock, usually in the setting of an acute coronary syndrome, characterised by hypotension, oliguria, and peripheral vasoconstriction

- acute worsening (decompensation) of CHF.

See end of article for authors' affiliations

\section{Correspondence to:} Dr Arend Mosterd, Department of Cardiology, Meander Medical Centre, PO Box 1502, 3800 BM Amersfoort, The Netherlands; a.mosterd@ meandermc.nl

\section{Systolic versus diastolic heart failure, impaired versus preserved left ventricular ejection fraction}

Heart failure traditionally was seen to result from impairment in ability of the heart to pump sufficient amounts of blood into the circulation during systole-that is, left ventricular systolic dysfunction. Echocardiography is most often employed to assess left ventricular systolic function, an ejection fraction of $\leqslant 40 \%$ indicating impaired left ventricular systolic function. Heart failure can also occur in patients with normal left ventricular systolic function in whom higher filling pressures are needed to obtain a normal end-diastolic volume of the left ventricle, so called heart failure with preserved left ventricular ejection fraction (PLVEF) or "diastolic" heart failure. ${ }^{\mathrm{W} 7}$ 
Establish the presence or absence of heart failure in patients suspected of the syndrome

Does my patient have heart failure (diagnosis)? (risk factors, incidence, prevalence)
No: prevention of heart failure (prevention)
Figure 1 The evaluation and management of heart failure.

Identify type of heart failure, cause and contributing factors

Why does my patient have (worsening) heart failure (aetiology)?

What will happen to my heart failure patient (prognosis)?

Initiate treatment

Can I improve the prognosis (ie reduce mortality, morbidity, hospitalisations, complaints or

increase quality of life) of my heart failure patient (therapeutic management)?

- Pharmacological

- Non-pharmacological: diet, fluid restriction, exercise, quit smoking

- Correct ischaemia, valvular heart disease, eliminate contributing factors (fever, use of non-steroida anti-inflammatory drugs, anaemia, alcohol excess, thyroid dysfunction, poor drug compliance, etc)

- Consider device therapy (implantable cardioverter-defibrillator and/or cardiac resynchronisation)

- Inform and instruct patient and his/her relatives

- Coordinate care

As the terms systolic and diastolic heart failure are not mutually exclusive-in heart failure patients with impaired left ventricular systolic function, diastolic abnormalities are often found as well-it is more appropriate to distinguish between heart failure with normal/preserved and impaired left ventricular ejection fraction. The occurrence of heart failure with PLVEF has been documented in numerous population-based studies, ${ }^{5}$ as well as in studies of patients presenting to the hospital with acute pulmonary oedema. ${ }^{\text {w8 }}$ Heart failure with PLVEF is more common in women, at a higher age, and in persons with longstanding hypertension, and carries a better prognosis than heart failure caused by impaired left ventricular systolic function. A recent hospital-based study among 2802 patients admitted with heart failure in Ontario, Canada, reported similar 1 year mortality rates in patients with preserved ejection fraction $(>50 \%)$ and those with an ejection fraction $<40 \%$ : $22 \%$ versus $26 \%(\mathrm{p}=0.07) .{ }^{\mathrm{w} 9}$ The hospital based nature of this study with its inherent biases of patient inclusion, selection for echocardiography and coding of heart failure is likely to account for the relatively poor prognosis in patients with preserved left ventricular function. Although the importance of heart failure with PLVEF is undisputed, there is no consensus on the exact definition of this entity and some researchers even argue that the prevalence of "diastolic heart failure" is grossly overestimated. ${ }^{\text {w10-12 }}$

\begin{tabular}{l}
$\begin{array}{l}\text { Table } 1 \text { European Society of Cardiology definition of heart } \\
\text { failure }\end{array}$ \\
\hline I. Symptoms of heart failure (at rest or during exercise) \\
and \\
II. Objective evidence (preferably by echocardiography) of cardiac \\
dysfunction (systolic and/or diastolic) (at rest) \\
and (in cases where the diagnosis is in doubt) \\
III. Response to treatment directed towards heart failure \\
\hline Criteria I and II should be fulfilled in all cases.
\end{tabular}

\section{Asymptomatic versus symptomatic left ventricular dysfunction}

Population-based echocardiographic studies have demonstrated that more than $50 \%$ of participants with left ventricular systolic dysfunction (generally defined as LVEF <35-40\%) have no symptoms or signs of heart failure. ${ }^{78 \mathrm{wl}}$ Asymptomatic left ventricular systolic dysfunction is found more frequently in person with coronary artery disease (relative risk (RR) 12.5, 95\% confidence interval (CI) 4.5 to 33.3), hypertension (RR 3.5, 95\% CI 1.4 to 8.5 ) or an abnormal ECG (RR 7.1, 95\% CI 2.8 to 16.7). Asymptomatic left ventricular dysfunction is an important precursor of heart failure and other cardiovascular events. ${ }^{9}$ ${ }^{\text {w14 }}$ By definition heart failure is characterised by symptoms and signs and as such asymptomatic left ventricular systolic dysfunction is not equivalent to heart failure.

\section{NYHA classification versus AHA/ACC staging}

The New York Heart Association (NYHA) classification has traditionally been used to classify severity of heart failure and indicate prognosis and, thus, to guide patient management. The severity of heart failure ranges from essentially asymptomaticwell treated patients in whom symptoms have been relieved (NYHA I) - to mild (NYHA II, slight limitation in physical activity), to moderate (NYHA III, symptoms while walking on

Table 2 Heart failure: symptoms and signs

\begin{tabular}{ll}
\hline Symptoms & Signs \\
\hline Dyspnoea (on exertion, nocturnal) & Oedema, ascites \\
Reduced exercise tolerance & Elevated jugular venous pressure \\
Fatigue, lethargy & Crepitations or wheeze \\
Orthopnoea & Tachycardia \\
Nocturnal cough & Third heart sound, murmurs \\
Wheeze & Hepatomegaly \\
Anorexia & Displaced apex beat \\
Confusion/delirium (elderly) & Cachexia and muscle wasting \\
\hline &
\end{tabular}


Table 3 American College of Cardiology/American Heart Association stages of heart failure ${ }^{w 15}$

\begin{tabular}{lll}
\hline Stage & Description & Example \\
\hline A & High risk, no symptoms & $\begin{array}{l}\text { Hypertension, coronary artery } \\
\text { disease, diabetes mellitus } \\
\text { Left ventricular hypertrophy, } \\
\text { asymptomatic left ventricular systolic } \\
\text { dysfunction }\end{array}$ \\
B $\quad \begin{array}{l}\text { Structural heart disease, no } \\
\text { symptoms }\end{array}$ & $\begin{array}{l}\text { Dyspnoea or fatigue due to heart } \\
\text { C frilure }\end{array}$ \\
D $\quad \begin{array}{l}\text { Structural heart disease, } \\
\text { Structural heart disease, } \\
\text { refractory symptoms }\end{array}$ & Patients with end stage heart failure \\
\hline
\end{tabular}

the flat), to severe heart failure (NYHA IV, breathless at rest and essentially housebound). An NYHA III class patient may improve to class II upon initiation of treatment, indicating that the NYHA classification is essentially a functional/symptomatic score, not taking into account the underlying cardiac disorder that will almost inevitably progress.

The staged American College of Cardiology/American Heart Association (ACC/AHA) heart failure classification acknowledges that heart failure is largely preventable (by control of blood pressure and other risk factors), is generally preceded by asymptomatic structural and functional cardiovascular abnormalities, and when present generally progresses (table 3). ${ }^{\text {w15 }}$ Neurohormonal activation, as measured by natriuretic peptide values, in persons without heart failure increases the risk of heart failure. ${ }^{\text {w16 }}$

Although the ACC/AHA classification emphasises the importance of recognising patients at high risk of developing heart failure and of correcting/treating major risk factors for the condition, the categorisation of these determinants-for example, diabetes or hypertension as "stage A heart failure"-is unjustified, if not misleading, since the criteria included in the definition of heart failure (that is, ventricular dysfunction and accompanying symptoms) are not fulfilled. For similar reasons, we prefer the term "asymptomatic left ventricular dysfunction" to the term "stage B heart failure".

\section{OCCURRENCE OF HEART FAILURE Heart failure in the real world}

Since the review by Cowie et al, ${ }^{1}$ which provides an extensive overview of studies published until 1995, numerous papers have been published addressing aspects of the heart failure epidemic from patients in population based cohorts to the highly selected groups of participants in clinical trials. The estimates vary considerably owing to a lack of uniformity in the definition and assessment of heart failure, that can be attributed to the absence of a gold standard for heart failure. ${ }^{\mathrm{w} 17}$ In addition, non-cardiac conditions (chronic obstructive pulmonary disease (COPD), obesity or a poor physical condition) may mimic heart failure. ${ }^{\text {w12 }}$ If heart failure is deemed present on clinical grounds (medical history, signs and symptoms) heart failure with PLVEF ("diastolic heart failure") is hard to tell apart from heart failure caused by impaired LVEF without the use of Doppler echocardiography or invasive measurements, although some features may point to heart failure with PLVEF (table 4). ${ }^{\text {w18 }}$

Ideally, estimates of the "true" prevalence and incidence of heart failure should be based on surveys in random samples of the general population, using validated questionnaires and properly conducted physical examinations (to document symptoms and signs of heart failure as well as medication use), and objective methods (echocardiography, electrocardiography, neurohormones) to demonstrate cardiac dysfunction, thus allowing an expert panel to classify patients as (not) having heart failure. In this way the presence of systolic and diastolic dysfunction in relation to the symptoms and signs of heart failure can be determined. Only a limited number of population based studies have used this approach.

The majority of patients with heart failure are managed by general practitioners rather than by cardiologists-the typical heart failure patient in general practice being an elderly woman with longstanding hypertension (more often suffering from "diastolic" heart failure)—whereas cardiologists tend to see men in their 60s who have had a myocardial infarction (having systolic heart failure). Heart failure patients in general practice are generally 15 years older (79 vs 64 years), more often women ( $58 \%$ vs $22 \%$ ), more often have (a history of) hypertension, and are less likely to have had a myocardial infarction (15\% vs $43 \%$ ) than heart failure patients seen by cardiologists. ${ }^{10}$ The average age of heart failure patients in general practice in the UK is 77 years. $^{\text {w19 }}$

These differences are important to bear in mind when interpreting the results of randomised clinical trials in heart failure; such trials have only recently begun to include patients older than 75-80 years and patients with heart failure with preserved left ventricular systolic function. ${ }^{\text {w20 }}$

\section{Prevalence and incidence of heart failure}

Generally speaking, the prevalence of heart failure can be estimated at $1-2 \%$ in the western world and the incidence approaches 5-10 per 1000 persons per year. Estimates of the occurrence of heart failure in the developing world are largely absent. ${ }^{11}$ w21

\section{Prevalence of heart failure}

Persons younger than 50 years are hardly ever found to have heart failure, but in those older than 50 years the prevalence and incidence increase progressively with age. In a recent US population-based study the prevalence of heart failure was $2.2 \%$ (95 CI $1.6 \%$ to $2.8 \%$ ), increasing from $0.7 \%$ in persons aged 45 through 54 years to $8.4 \%$ for those aged 75 years or older. ${ }^{8}$ Congestive heart failure was found to be more common in women. These figures are highly comparable to previous studies, such as the Rotterdam study (prevalence of heart failure being $1 \%$ in age group 55-64 years, 3\% in age group 6574 years, $7 \%$ in age group $75-84$ years, and over $10 \%$ in those aged $\geqslant 85$ years $)$. ${ }^{\text {13 }}$

The first prevalence study to use two-dimensional echocardiography in the population at large took place in Glasgow ( 1647 participants, 48\% men, mean age 50 years), deeming heart failure present if LVEF was $<30 \%$ in persons having cardiac shortness of breath or using loop diuretics. The prevalence of heart failure was $1.5 \%$ (1.6\% in men, $1.4 \%$ in women). Interestingly asymptomatic left ventricular systolic dysfunction was found to occur very frequently (overall $1.4 \%$, men $2.4 \%$, women $0.5 \%$ ). The Rotterdam study (using M-mode echocardiography in a subgroup of 2267 participants, aged 55 years or older) also found left ventricular systolic dysfunction to 
Table 4 Findings suggestive of diastolic heart failure ${ }^{\mathrm{w} 18}$

- Pronounced hypertension during episode of heart failure (systolic blood pressure $>160 \mathrm{~mm} \mathrm{Hg}$, diastolic blood pressure $>100 \mathrm{~mm} \mathrm{Hg}$ )

- Echocardiography: concentric left ventricular hypertrophy without wall motion abnormalities, increased relative wall thickness, normal end diastolic volume

- Tachycardia with a shortened diastolic filling period (for example, rapid atrial fibrillation)

- Precipitation of heart failure by the infusion of a small amount of intravenous fluid

- Clinical improvement in response to treatment directed at the cause of diastolic dysfunction (such as lowering blood pressure, reducing heart rate, or restoring the atrial "kick")

occur more frequently in men than in women $(5.5 \%$ vs $2.2 \%) .{ }^{w 13}$ Subsequent studies have confirmed these earlier data and confirmed that many persons (more than half) with impaired left ventricular systolic function have no symptoms or signs of heart failure at all. ${ }^{8}$

Only a few epidemiological studies have used echocardiography to investigate diastolic dysfunction specifically, other studies defining diastolic heart failure as symptoms and signs of heart failure in the presence of a normal left ventricular ejection fraction (that is heart failure with preserved left ventricular systolic function). ${ }^{568}$ w22 w23 The MONICA Augsburg study (1274 persons, aged 25 to 75 years) found a high prevalence of diastolic function abnormalities, assessed by Doppler echocardiography, increasing from $2.8 \%$ in persons aged 25-35 years to $15.8 \%$ in those over 65 years. $^{\text {w23 }}$ Interestingly, in participants without left ventricular hypertrophy, hypertension, coronary artery disease, obesity or diabetes, diastolic function abnormalities were largely absent.

The most all inclusive epidemiologic study of heart failure prevalence in the population is from Olmsted County, Minnesota, USA. $^{8}$ In 2042 persons older than 45 years the presence of heart failure was established and Doppler echocardiography was used to assess left ventricular systolic and diastolic dysfunction in great detail. In addition to measuring standard transmitral flow parameters, pulmonary venous flow, mitral inflow at peak Valsalva manoeuvre and Doppler tissue imaging of mitral annular motion were used to characterise diastolic function. The prevalence of heart failure was $2.2 \%$, $44 \%$ having an ejection fraction higher than 50\%; $7.3 \%$ were found to have moderate or severe diastolic dysfunction, generally $(75 \%)$ with normal ejection fraction. Moderate to severe systolic dysfunction (ejection fraction $<40 \%$ ) was found in $2.0 \%$. Less than half of those having moderate or severe diastolic or systolic dysfunction had heart failure.

\section{Incidence of heart failure}

Reliable estimates of the incidence of heart failure are available from the Rotterdam and Hillingdon heart failure studies. ${ }^{\text {w24 w25 }}$ Both studies are population based and used an expert panel to establish the presence or absence of heart failure. In the Hillingdon study the incidence of heart failure increased from $0.2 / 1000$ person years in those aged $45-55$ years to $12.4 / 1000$ person years in those aged $>85$ years. In Rotterdam the incidence increased from 2.5/1000 person years (age 5564 years) to $44 / 1000$ person years ( $>85$ years or older). Heart
- Prevalence: The number of persons having heart failure at a certain moment in time (generally expressed as a percentage).

- Incidence: The number of persons newly diagnosed with heart failure during a certain time period (generally expressed as the number of new cases of heart failure per 1000 persons per year - the incidence rate-and sometimes expressed as a proportion of those free from the disease that develops the disease within a specified time period-for example, the 5 year cumulative incidence).

failure occurs more frequently in men than in women (15 and 12 per 1000 person years, respectively). The higher figures in Rotterdam most probably reflect differences in methodology (including evaluation of all patients being prescribed diuretics or angiotensin converting enzyme (ACE) inhibitors in Rotterdam) rather than true differences in the incidence of heart failure. Interestingly, a more recent analysis from the Rotterdam study, although using a somewhat different methodology to identify incident heart failure, produced remarkably similar results. ${ }^{\text {w26 }}$

\section{Hospitalisations for heart failure}

The number of hospitalisations for heart failure (usually expressed as a number per 1000 patients per year) and the change in this measure provide useful information on the epidemic of heart failure. It should be emphasised, however, that hospitalisation rates result from a complex interaction of multiple determinants, including the prevalence, incidence and survival of the disease, referral patterns and treatment possibilities in primary care as well as discharge diagnosis coding practices. Consequently, time trends in hospitalisation rates are often difficult to interpret.

Age adjusted hospitalisation rates increased considerably throughout the western world in the 1980s and early 1990s, as documented by reports from New Zealand, the USA, Sweden, Scotland, and the Netherlands. ${ }^{1}{ }^{\text {w27 }}$ Recent reports have suggested that admission numbers have peaked in the 1990s (at least in Scotland, the Netherlands and Sweden) and that the prognosis of hospitalised heart failure patients has improved (fig 2). ${ }^{12}$ w28-30

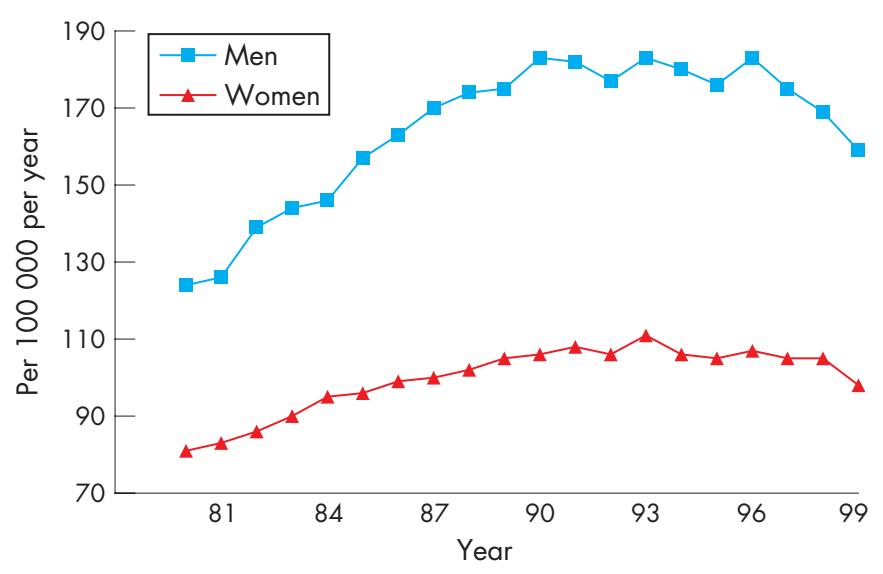

Figure 2 Admissions for heart failure in Dutch hospitals, 19801999. ${ }^{\mathrm{w} 29}$ 
In the Netherlands the total number of hospitalisations for heart failure increased by 72\% between 1980 ( 14 441) and 1999 (24 868). Part of this increase is explained by the ageing of the population, the mean age of patients admitted with heart failure increasing from 71.2 to 72.9 years in men and from 75.0 to 77.7 years in women during the same period. After reaching a peak in 1992 (men) and 1993 (women), age adjusted hospitalisation rates for heart failure started to decline by $1.0-1.5 \%$ per year. Concomitantly, the average duration of hospitalisation declined from 21.1 days in 1980 to 12.9 days in 1999. In-hospital mortality declined from $18.6 \%$ to $13.5 \%$. ${ }^{\text {w29 }}$

One hospital based study specifically addressed secular trends in prevalence and prognosis of heart failure patients with or without PLVEF. ${ }^{13}$ The prevalence of heart failure with PLVEF in patients admitted to the Mayo Clinics increased from $38 \%$ to $54 \%$ between 1987 to 2001 , concomitant with an increase in prevalence of hypertension, atrial fibrillation and diabetes. In this time frame survival improved significantly for patients with impaired left ventricular systolic function, but did not change in those with preserved left ventricular systolic function.

In the face of the predicted increase in age adjusted prevalence of heart failure, ${ }^{14}$ the observed decline in hospitalisation rates may come as a surprise. However, hospitalisations for heart failure do not reflect the occurrence and prognosis of heart failure in the community, as they relate only to the more severe stages of heart failure warranting in-hospital evaluation and treatment. The decline in hospitalisation rates may well be due to improved treatment and management of heart failure patients-for example, by means of dedicated heart failure clinics. In addition, it is conceivable that an increasing number of patients with terminal heart failure are being cared for in a home-based setting by their general practitioner, rather than being admitted to hospital.

\section{Aetiology and risk factors for heart failure Aetiology of heart failure}

Although many conditions can cause heart failure (coronary artery disease, hypertension, cardiomyopathies, valvular and congenital heart disease, arrhythmias, pericardial disease, myocarditis, pulmonary hypertension, and cardiotoxic substances-including alcohol), the predominant cause of heart failure in the western world is ischaemic heart disease (table 5). ${ }^{15} 16^{\mathrm{w} 24}$ The variation in frequencies of causes of heart failure reported in different studies can be explained by differences in study population, from the highly selected group of participants in clinical trials to relatively unselected participants in population-based studies, differences in definitions, and time differences (the Framingham heart study originated in 1948). In addition, it has become clear that using non-invasive methods the precise aetiology of heart failure cannot always be determined accurately. In the Bromley heart failure study the percentage of heart failure with unknown cause declined from $42 \%$ to $10 \%$ after nuclear testing and cardiac catheterisation, while the percentage of patients with ischaemic heart failure increased from $29 \%$ to $52 \%{ }^{16}$

\section{Risk factors for heart failure}

Various (population-based) studies have addressed risk factors for the occurrence of heart failure. ${ }^{15} 17$ w31-34 Coronary artery disease notably increases the chance of developing heart failure; in 7-8 years after myocardial infarction up to $36 \%$ of patients will experience heart failure, especially those with left ventricular systolic dysfunction documented during admission. ${ }^{18}$

Although the risk of heart failure associated with hypertension (systolic blood pressure $>140 \mathrm{~mm} \mathrm{Hg}$, or diastolic blood pressure $>90 \mathrm{~mm} \mathrm{Hg}$, or treatment with antihypertensive medication) is smaller than that associated with myocardial infarction, hypertension contributes considerably to the population burden of heart failure as it occurs more frequently than myocardial infarction (table 6$).{ }^{15}$ Obesity (body mass index $>30 \mathrm{~kg} / \mathrm{m}^{2}$ ), increasingly present in western societies, doubles the risk of heart failure after adjustment for associated risk factors. ${ }^{19}$ Valvular abnormalities, factors indicative of heart disease (left ventricular hypertrophy, left ventricular dilatation), a parental history of heart failure, conventional risk factors (such as smoking, diabetes, obesity), as well extracardiac conditions (renal dysfunction, obstructive pulmonary disease) all increase the risk of heart failure (table 6). ${ }^{17}$ w35 w36

The relation of obesity, increased cholesterol values and hypertension to cardiovascular morbidity and mortality is undisputed. In patients with CHF considerable evidence exists that obesity, hypercholesterolaemia and hypertension are "protective". This phenomenon has been termed "reverse epidemiology" for which a comprehensive explanation is still lacking..$^{20}$ Proposed explanations are the syndrome of cardiac cachexia, reverse causation (the underlying cause of hypotension-that is, pump failure-being detrimental rather than hypotension per se) and time discrepancies among competitive risk factors. The reverse epidemiology does not hold for all conventional risk factors, as quitting smoking is associated with a better prognosis in heart failure patients. ${ }^{21}$

\section{Comorbidity in heart failure}

Heart failure, being a common disease in the elderly, should not be viewed in isolation: anemia, cachexia, renal impairment, obstructive sleep apnoea, chronic pulmonary disease and diabetes mellitus are conditions frequently observed in heart failure patients and unfavourably affect prognosis. ${ }^{2}$ Comorbidity was found to be one of the prime determinants of prognosis in a study of patients admitted with heart failure and contributes to the poor quality of life as perceived by heart failure patients. ${ }^{\mathrm{W} 37 \mathrm{w} 38}$ The complex interplay between diseases previously perceived as entities on their own, such as COPD, renal disease, obstructive sleep apnoea on the one hand and heart failure on the other, is increasingly acknowledged.

Patients with chronic kidney disease constitute a group of patients at high risk for having or developing cardiovascular disease, including heart failure. ${ }^{\text {w39 }}$ Heart failure in these patients may result from coronary heart disease, longstanding hypertension resulting in concentric hypertrophy, or volume overload due to anaemia, fluid overload and arteriovenous fistulas leading to left ventricular dilatation. The prevalence of heart failure increases with severity of renal impairment; approximately $20 \%$ of patients with a glomerular filtration rate $<30 \mathrm{ml} / \mathrm{min} / 1.73 \mathrm{~m}^{2}$ (not on dialysis) have heart failure. ${ }^{\mathrm{w} 40 \mathrm{w} 41}$ Along similar lines, heart failure appears to be frequently present in patients with COPD; in a recent Dutch study of 405 patients older than 65 years with a diagnosis of COPD, $20.5 \%$ were found to have previously unrecognised heart failure. ${ }^{22}$ 
Table 5 Causes of heart failure in population based studies

\begin{tabular}{|c|c|c|c|c|}
\hline \multirow[b]{2}{*}{ Cause } & \multicolumn{2}{|c|}{$\begin{array}{l}\text { Framingham heart } \\
\text { study }^{15}\end{array}$} & \multirow{2}{*}{$\begin{array}{l}\text { Hillingdon } \\
\text { heart failure } \\
\text { studyw24 }\end{array}$} & \multirow{2}{*}{$\begin{array}{l}\text { Bromley } \\
\text { heart } \\
\text { failure } \\
\text { study }\end{array}$} \\
\hline & Men & Women & & \\
\hline Ischaemic & 59 & 48 & 36 & 52 \\
\hline \multicolumn{5}{|l|}{ Non-ischaemic: } \\
\hline Hypertension & 70 & 78 & 14 & 4 \\
\hline Válvular heart disease & 22 & 31 & 7 & 10 \\
\hline Atrial fibrillation & & & 5 & 3 \\
\hline Alcohol & & & & 4 \\
\hline Other & 7 & 7 & 4 & 5 \\
\hline Unknown & & & 34 & 23 \\
\hline
\end{tabular}

Because of rounding, the percentages do not always add up to 100 . Framingham heart study: ischaemic heart disease and hypertension could be co-named as causing heart failure.

\section{Trends in heart failure incidence}

Bonneux et al predicted a steady increase in the number of patients with heart failure: the ageing of the population, improvements in the treatment of acute coronary syndromes, and a longer survival of heart failure patients all contribute to a larger pool of (potential) heart failure patients. ${ }^{14}{ }^{\mathrm{w}} 42$ The ageing of the population is undisputed as is the improvement of prognosis in heart failure patients.

Few studies have addressed trends in the incidence of heart failure post-myocardial infarction. Despite a decrease in coronary heart disease and all cause mortality in 546 Framingham heart study participants who suffered a non-Q wave myocardial infarction between 1950 and 1989, the percentage of them developing heart failure remained stable. $^{\text {w43 }}$ In a group of 1537 patients who suffered a myocardial infarction (not excluding non-Q wave myocardial infarctions) between 1979 and 1994 in Olmsted County, Minnesota, a $28 \%$ reduction in the occurrence of postmyocardial infarction heart failure was documented. ${ }^{18}$ The Worcester heart attack study reported a decline in heart failure during hospitalisation for myocardial infarction between 1975 and $1995 .^{\text {w44 }}$

The reduction of post-myocardial infarction heart failure is consistent with the declining severity of myocardial infarction following the introduction of reperfusion treatment-a decline that may well continue given the increasingly aggressive (primary percutaneous interventions) and timely interventions in patients with acute coronary syndromes. ${ }^{\text {W45 }}$ These data suggest that improved survival following myocardial infarction is not a major contributor to the occurrence of heart failure.

The incidence of heart failure in men participating in the Framingham heart study did not change over the last 50 years (1950-1999), whereas the incidence in women declined 30$40 \%{ }^{\text {w46 }}$ A larger population-based study in Olmsted County, Minnesota (4537 heart failure patients, $42 \%$ of whom were diagnosed as outpatients) reported no change in heart failure incidence between 1979 and 2000. ${ }^{23}$

Taken together, the data indicate that the incidence of heart failure has not declined over the last two decades and that the ageing of the population in combination with improved prognosis fuel the heart failure epidemic. It follows that prevention of the occurrence of heart failure is needed to stem the epidemic. ${ }^{\mathrm{w} 42}$

\section{PROGNOSIS OF HEART FAILURE \\ "A poor prognosis" and prognostication in daily practice}

There is no doubt that the prognosis of heart failure patients remains poor, even in the realm of the development of a myriad of effective pharmacological and non-pharmacological interventions. This is illustrated by the title of a paper on the prognosis of the syndrome: "More malignant than cancer". ${ }^{\text {w47 }}$ Any doctor treating heart failure patients will confirm that life expectancy in heart failure patients is "reduced" and that sudden cardiac death is a "major" cause of death, that (acute) worsening of CHF occurs "quite often", leading to "frequent" hospitalisations, and that quality of life in these patients is "impaired considerably". We included the quotation marks in the latter sentence to indicate the implicit nature of prognostication in clinical practice.

Although information on the natural history of a disease is relevant to illustrate its burden for health care and the society at large, prognostication in individual patients plays a crucial role in daily clinical practice. After the diagnosis (and possible aetiology) of heart failure has been established, a doctor will estimate an individual patient's probability of developing clinically relevant prognostic outcomes-for example, a 5 year survival probability. Such estimates are typically based on patients' characteristics, including age, comorbidity, severity and cause of heart failure that are known to influence prognosis. This information, together with the anticipated, preferably evidence-based, effect of possible therapeutic interventions and patient preferences, is instrumental in the

Table 6 Risk factors for the occurrence of heart failure in three population based studies ${ }^{15}$ w13 w31

\begin{tabular}{|c|c|c|c|c|c|c|c|c|}
\hline \multirow[b]{3}{*}{ Risk factor } & \multicolumn{4}{|c|}{ Framingham heart study } & \multirow{2}{*}{\multicolumn{2}{|c|}{$\begin{array}{l}\text { Cardiovascular health } \\
\text { study }\end{array}$}} & \multicolumn{2}{|l|}{ Rotterdam study } \\
\hline & \multicolumn{2}{|l|}{ Men } & \multicolumn{2}{|l|}{ Women } & & & \multirow{2}{*}{$\begin{array}{l}\text { Men } \\
\text { RR }(95 \% \mathrm{Cl})\end{array}$} & \multirow{2}{*}{$\begin{array}{l}\text { Women } \\
\text { RR }(95 \% \mathrm{Cl})\end{array}$} \\
\hline & $\operatorname{RR}(95 \% \mathrm{Cl})$ & PAR & $\operatorname{RR}(95 \% \mathrm{Cl})$ & PAR & $\mathbf{R R}$ & PAR & & \\
\hline Hypertension & 2.1 (1.3 to 3.2 ) & 39 & 3.4 (1.7 to 6.7$)$ & 59 & 1.4 & 13 & $1.0(0.5$ to 1.9$)$ & 2.6 (1.6 to 4.2$)$ \\
\hline $\mathrm{Ml}$ & $6.3(4.6$ to 8.7$)$ & 34 & 6.0 (4.4 to 8.3$)$ & 13 & - & - & $1.9(1.1$ to 3.6$)$ & $1.8(0.9$ to 3.5$)$ \\
\hline Angina pectoris & $1.4(1.0$ to 2.0$)$ & 5 & $1.7(1.2$ to 2.3$)$ & 5 & - & - & $1.3(0.6$ to 2.8$)$ & $1.3(0.7$ to 2.6$)$ \\
\hline Diabetes mellitus & $1.8(1.3$ to 2.6$)$ & 6 & 3.7 (2.7 to 5.2$)$ & 12 & 1.8 & 8 & $2.1(1.0$ to 4.4$)$ & 1.6 (0.8 to 3.2$)$ \\
\hline LVH & $2.2(1.5$ to 3.2$)$ & 4 & $2.9(2.0$ to 4.1$)$ & 5 & 2.3 & 6 & $1.6(0.4$ to 6.7$)$ & 0.8 (0.1 to 5.5$)$ \\
\hline Valvular disease & 2.5 (1.7 to 3.6$)$ & 7 & $2.1(1.5$ to 2.9$)$ & 8 & - & - & - & - \\
\hline Atrial fibrillation & & & & & 2.1 & 2 & $1.5(0.5$ to 5.1$)$ & $0.6(0.1$ to 4.6$)$ \\
\hline COPD & & & & & 1.4 & 6 & $0.8(0.3$ to 2.6$)$ & $3.2(1.7$ to 7.4$)$ \\
\hline
\end{tabular}

$\mathrm{Cl}$, confidence interval; COPD, chronic obstructive pulmonary disease; LVH, left ventricular hypertrophy; Ml, myocardial infarction; PAR, population attributable risk (\%); $\mathrm{RR}$, relative risk. 
decision which therapeutic measures should be taken. Importantly, this implies precise enough predictions of the future-a difficult task, the more since, apart from survival, other prognostic outcomes are of interest in heart failure, including hospitalisations and quality of life. Moreover, and understandably, practising physicians seem reluctant to communicate quantitative prognostic information explicitly (for example, "your probability of surviving the next 5 years is $40 \%$ ") to their patients, ${ }^{\text {w48 }}$ although the number of studies identifying prognostic factors and quantifying its predictive potential is rapidly increasing. The recent development of prognostic scores, enabling physicians to estimate an individual's probability of developing relevant complications as a function of the level of a limited number of prognostic factors, may be helpful.

\section{Mortality and causes of death}

The best impression of the prognosis of the "average" heart failure patient is obtained in population based research, in which incident cases of established heart failure are followed up carefully. Such studies have been carried out in the USA, UK,

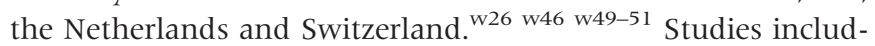
ing incident cases admitted to hospital show that the mortality rate is relatively high in the first few weeks after the occurrence of heart failure, while after that period the slope of the survival curve shows a much more gradual slope. The 30 day mortality is reported to lie around $10-20 \%$. Both in the US (Framingham heart study) and in England (Hillingdon heart failure study) 1 year survival following the onset of heart failure was $70 \%$. Five years after the occurrence of heart failure only $35 \%$ of Framingham participants were still alive. Survival rates in a study on prevalent cases of heart failure from the Netherlands (Rotterdam study) were more favourable: 1, 2 and 5 year survival rates of $89 \%, 79 \%$ and $59 \%$, respectively, reflecting a threefold increase in the risk of death compared to the age and gender matched population at large. ${ }^{\mathrm{w} 52}$ The 1 year survival rate in the study from Switzerland was $77 \% .{ }^{\text {w51 }}$ These differences are most likely to be attributable to differences in patient selection and definition of heart failure applied, leading to more patients with mild heart failure in the Rotterdam study.

A more recent report from the Rotterdam study including cases of incident heart failure, and allowing for inclusion of patients hospitalised for heart failure as incident cases, produced lower 1, 2 and 5 year survival rates of $63 \%, 51 \%$ and $35 \%$, respectively. ${ }^{\mathrm{w} 26}$ The importance of the disease spectrum is further illustrated by the comparison with survival rates from hospitalised-based studies. A large hospital-based study from Leicestershire (12 220 index admissions, 19932001 ) reported a very low 1 year survival rate of $57 \% .{ }^{\text {w53 }}$ In contrast, survival rates from (placebo-treated) participants from the large randomised trials tend to underestimate mortality, since participants in these trials are usually not representative of the "average" heart failure patient. For example, a meta-analysis of $\beta$-blockers in heart failure found a 1 year mortality in placebo recipients of "only" $12 \%$ in trials carried out in the late 1990s. ${ }^{\text {w54 }}$ Apart from differences in age, gender and type of heart failure, the poor prognosis in the general population can also be attributed to the impressive comorbidity in many patients with heart failure. Anaemia, cachexia, renal impairment, obstructive sleep apnoea, COPD
Table 7 Age adjusted mortality (\%) after onset of heart failure in men and women aged 65-74 years in the Framingham heart study during the period 1950-1999

\begin{tabular}{lrrrr}
\hline Period & $\begin{array}{l}\mathbf{1} \text { year } \\
\text { mortality in } \\
\text { men }\end{array}$ & $\begin{array}{l}\mathbf{1} \text { year } \\
\text { mortality in } \\
\text { women }\end{array}$ & $\begin{array}{l}\mathbf{5} \text { year } \\
\text { mortality in } \\
\text { men }\end{array}$ & $\begin{array}{l}\mathbf{5} \text { year } \\
\text { mortality in } \\
\text { women }\end{array}$ \\
\hline $1950-1969$ & 30 & 28 & 70 & 57 \\
$1970-1979$ & 41 & 28 & 75 & 59 \\
$1980-1989$ & $33(30)$ & $27(20)$ & $65(65)$ & $51(51)$ \\
$1990-1999$ & $28(21)$ & $24(17)$ & $59(50)$ & $45(46)$ \\
\hline
\end{tabular}

Data in parentheses are from Olmsted County ( 4537 heart failure patients with a mean age of 79 years for women and 73 years for men). ${ }^{23}$ w46

and diabetes mellitus are conditions frequently observed in heart failure patients and unfavourably affect prognosis. ${ }^{2}$

The vast majority of patients with heath failure die from cardiovascular causes. Estimates vary from 50-90\%, depending, again, on the heart failure population studied. The assessment of the cause of death in heart failure patients often poses difficulties. Frequently a distinction is made between sudden or unexpected cardiac death, death due to progressive heart failure, and death due to other causes. In the ATLAS trial (high versus low dose lisinopril in NYHA functional class II to IV heart failure patients) mode of death in 1381 patients was classified as follows: 589 (43\%) sudden death, 443 (32\%) progressive heart failure, and 349 (25\%) due to other causes. ${ }^{\text {w55 }}$ Especially sudden cardiac death poses a major threat. Some studies report that up to $50 \%$ of heart failure patients die suddenly, but because of large differences in the definition of sudden cardiac death, and the lack of consensus on this, the exact magnitude of the problem is unknown.

Some studies indicate patients with relatively mild heart failure are more susceptible to arrhythmias and sudden cardiac death, while patients in more advanced stages of the disease (NYHA III and IV) often die from end stage ventricular dysfunction. In view of the large proportion of patients suffering from major cardiac arrhythmias and sudden cardiac death, and the development of effective-but often expensivetargeted therapeutic interventions such as implantable cardioverter-defibrillators and pacing devices, identification of those heart failure patients most likely to develop this fatal complication is an important clinical challenge. This requires knowledge on predictors of sudden cardiac death and preferably the development of algorithms combining several predictors readily available in daily practice, capable of estimating an individual patient's risk of sudden death.

\section{Trends in mortality}

The prognosis of patients with heart failure in the population is improving. In the Framingham heart study, the 1 and 5 year mortality rates from heart failure in men declined from $30 \%$ and $70 \%$ in the period 1950 to 1969 to $28 \%$ and $59 \%$ in the period 1990 to 1999 . In women, 1 year mortality rates decreased from $28 \%$ to $24 \%$ and the 5 year mortality rates decreased from $57 \%$ to $45 \%$ during the same period (table 7 ). ${ }^{\mathrm{w} 46}$ These results are confirmed by a larger population-based study in Olmsted County, Minnesota, that demonstrated an improvement in prognosis, particularly in men and younger heart failure patients (table 7). ${ }^{23}$ Similarly, a report from the Hillingdon heart failure studies documented a decrease in 
Table 8 Non-exhaustive list of parameters associated with the prognosis of heart failure patients

\begin{tabular}{llll}
\hline Patient characteristics/comorbidity & $\begin{array}{l}\text { Functional parameters and } \\
\text { ventricular function indices }\end{array}$ & Laboratory measurements & Interventions received \\
\hline Age & NYHA class & B-type natriuretic peptide & ACE inhibition \\
Gender & 6 min walk test & Cholesterol & $\beta$-blockers \\
Aetiology of HF, eg, myocardial ischaemia & Ejection fraction & Heart rate & Spironolactone \\
Diabetes & Ventricular mass & QRS duration & Statins \\
Renal dysfunction & & Cardiothoracic ratio & \\
Anaemia & & Haemoglobin & \\
Depression & Creatinine & \\
\hline
\end{tabular}

ACE, angiotensin converting enzyme; HF, heart failure; NYHA, New York Heart Association.

6 month mortality in heart failure patients from $25 \%$ (1995) to 14\% (2005) (PA Mehta et al, presented at the European Society of Cardiology meeting, Barcelona, September 2006).

\section{Determinants of prognosis in heart failure}

Many determinants of the prognosis in heart failure have been identified (table 8). It is important to note that in order to be of importance and serve practice, prognostic determinants need not be causally related to the prognostic outcome. Age and gender, for example, are important prognostic markers in many diseases, even after adjustment for other prognostic determinants, although age per se may not be causally implicated, but indicates other, often immeasurable, factors that are aetiologically involved. Prognostic determinants in heart failure can be arbitrarily categorised in: (1) patient characteristics and comorbidity; (2) laboratory measurements; (3) functional parameters and ventricular function; and (4) interventions received. ${ }^{24} \mathrm{w} 56$

Apart from age, the NYHA classification has long been recognised as an important indicator of the severity of heart failure and, thus, survival. The prognosis of heart failure obviously relates to the cause of heart failure; patients who suffer heart failure caused by viral myocarditis may completely recover, while in patients with an acute myocardial infarction complicated by heart failure 1 year mortality exceeds $50 \%$. Comorbidities known to influence survival unfavourably in heart failure include renal dysfunction, depression and anaemia. Importantly, and for the reasons outlined above, this does not imply that correction, if possible, of these prognostic determinants improves survival. Patients with both heart failure and chronic renal failure have an extremely poor prognosis; this pathophysiological condition has been termed severe cardiorenal syndrome, in which combined cardiac and renal failure amplify progression of the individual organ pathology. ${ }^{\text {w57 }}$ In practice, not all these prognostic parameters will be known or even necessary. The combination of a few independent prognostic variables may be sufficient to guide patient management.

\section{Prognostic models}

Predicting the prognosis ("prognostication") in an individual patient with heart failure is instrumental in the decision to initiate or refrain from possible interventions. As explained above, the prognosis-for example, 5 year survival-of a particular patient is usually made implicitly on the basis of patient characteristics and available (for example, biochemical, haemodynamic, echocardiographic or electrocardiographic) measurements. By using a combination of those variables that independently predict prognosis, the absolute probability of an individual patient to develop the prognostic outcome of interest (for example, 1 year probability of survival) can be estimated by using mathematical modeling techniques. By means of multiple logistic regression and receiver operating characteristic (ROC) analyses a prognostic model or simplified score can derived, that can aid physicians in stratifying patients according to their prognosis. The derivation of a prognostic score requires a cohort of a sufficiently large number of representative patients with heart failure followed-up for a certain period, and in whom the occurrence of the prognostic outcome is monitored carefully. Before such a score can be applied in routine clinical practice, it should be carefully validated in other heart failure populations. Ideally, such a prognostic score includes only a limited number of readily, preferably routinely, available, cheap and nonpatient burdening parameters. Examples of prognostic scores that have been applied successfully in daily practice include the Apgar score in neonates and the Framingham risk function, predicting the occurrence of future cardiovascular events on the basis of an individual's risk factor level.

An example of a prognostic score is the one developed by Bouvy et al. ${ }^{\text {w5 }}$ In a study among 152 patients admitted for heart failure they developed a score, including eight items, to predict 18 month mortality (table 9). For example, a 60-yearold woman weighing $70 \mathrm{~kg}$, with a history of renal insufficiency, diabetes, ankle oedema, and a blood pressure of 130/ $80 \mathrm{~mm} \mathrm{Hg}$, who is not on a $\beta$-blocker, has a score of (60/ $17+0+9+17+10-70 / 3+0+13)=29.2$, reflecting an 18 month mortality of $78 \%$.

Several other prognostic scores are available. ${ }^{\text {w59 }}{ }^{\text {w60 }}$ Very recently, follow up data from the 1125 participants included in the PRAISE trial were used to develop models to predict 1,2 and 3 year mortality. ${ }^{24}$ This Seattle heart failure model was validated extensively in five other cohorts with a total of 9942 patients with heart failure. The model performed very well in these other cohorts. For example, the 1 year predicted survival using the model for the four validation cohorts were $90.5 \%$, $86.5 \%, 83.8 \%, 91.0 \%$ and $89.0 \%$, while the actual survival rates were remarkably similar at $88.5 \%, 86.5 \%, 83.3 \%, 91.0 \%$ and $86.7 \%$, respectively. A disadvantage of this score is that it includes as many as 14 continuous variables (including age, systolic blood pressure, weight, ejection fraction, uric acid and haemoglobin values) and 10 categorical variables, including gender, NYHA class, ischaemic aetiology, and indicators of the use of medications such as ACE inhibitors, $\beta$-blockers, statins, and devices. A web-based calculator is available at www.seattleheartfailuremodel.org. 
Table 9 Independent predictors (and their corresponding score) of 18 month mortality in patients admitted to hospital because of heart failure ${ }^{\text {w58 }}$

\begin{tabular}{|c|c|c|c|}
\hline Predictor & Score & Point score & $\begin{array}{l}18 \text { month } \\
\text { mortality }\end{array}$ \\
\hline Age (per year) & 0.06 & & \\
\hline Male sex & 4 & $<-15$ & $12 \%$ \\
\hline Diabetes & 9 & $\geqslant-15$ and $<-5$ & $10 \%$ \\
\hline Renal dysfunction & 17 & $\geqslant-5$ and $<-1$ & $8 \%$ \\
\hline Ankle oedema & 10 & $\geqslant-1$ and $<7$ & $46 \%$ \\
\hline Weight (per kg) & -0.4 & $\geqslant 7$ and $<11$ & $52 \%$ \\
\hline Low blood pressure & 7 & $\geqslant 11$ & $78 \%$ \\
\hline No use of $\beta$-blockers & 13 & & \\
\hline
\end{tabular}

*Systolic pressure $<110 \mathrm{~mm} \mathrm{Hg}$ or diastolic pressure $<70 \mathrm{~mm} \mathrm{Hg}$

Another prognostic score was developed for primary care patients. This may be important, since prognostic models developed in hospital patients or those included in large randomised trials may not be applicable to primary care with its different case mix of heart failure patients with possibly different prognostic markers. The primary care score included six items only (age, sex, B-type natriuretic concentration, history of stroke, diabetes and ECG abnormalities). ${ }^{\text {w61 }}$

Recently, a prognostic model was developed to predict the probability of sudden cardiac death. The analyses identified increased cardiothoracic ratio, QRS dispersion, QTc dispersion and non-sustained ventricular tachycardia as independent predictors of sudden death and these were combined in a score. Such a risk score could be useful in targeting preventive interventions, including relatively expensive device technology, at those patients most likely to benefit. w62 w63

\section{PREVENTION}

\section{Lifetime risk of heart failure}

The incidence figures from the Framingham heart study and Rotterdam study have been used to provide estimates of the life time risk of developing heart failure (table 10). ${ }^{25}{ }^{\mathrm{w} 26}$ The overall chance that a 40 -year-old person develops heart failure during the rest of his/her life is $21 \%$. In hypertensive persons (systolic blood pressure $>160 \mathrm{~mm} \mathrm{Hg}$ and/or diastolic blood pressure $>100 \mathrm{~mm} \mathrm{Hg}$ ) this chance is appreciably higher $(28 \%)$ than in normotensive persons; they have a lifetime heart failure risk of $13 \%$.

\section{Prevention of heart failure}

As coronary artery disease and hypertension are the predominant causes of heart failure, prevention of the onset of hypertension and coronary artery disease is key to reducing the burden of heart failure. ${ }^{\mathrm{w} 64}$ Given the high prevalence of hypertension in western societies, the impact of antihypertensive treatment may well be larger than that of adequate treatment of acute coronary syndromes (table 11). ${ }^{15}$ w65 w66

Patients at a high risk of developing heart failure, whether or not already having structural heart disease (such as left ventricular hypertrophy or asymptomatic left ventricular systolic dysfunction), benefit from treatment. Asymptomatic left ventricular systolic dysfunction predisposes to the occurrence of heart failure. ${ }^{914}$ During the 3 year follow up of 2117 participants randomised to placebo in the SOLVD prevention study (asymptomatic left ventricular dysfunction, ejection fraction $\leqslant 35 \%)$, $334(15.8 \%)$ participants died, $640(30.2 \%)$
Table 10 Five year and lifetime risk of developing heart failure in participants of the Framingham heart study and the Rotterdam study ${ }^{25}$ w 26

\begin{tabular}{llll}
\hline Age (years) & $\begin{array}{l}\mathbf{5} \text { year risk } \\
\text { (\%) }\end{array}$ & $\begin{array}{l}\text { life time risk } \\
\text { (\%) }\end{array}$ & $\begin{array}{l}\text { Life time risk in } \\
\text { hypertensive } \\
\text { persons* }\end{array}$ \\
\hline Men & & & \\
40 & $0.2 \%$ & $21 \%$ & 28 \\
$50(55)$ & $0.8 \%(0 \%)$ & $21 \%(33 \%)$ & 27 \\
$60(65)$ & $1.3 \%(4.2 \%)$ & $21 \%(33 \%)$ & 29 \\
$70(75)$ & $4.0 \%(9.5 \%)$ & $21 \%(30 \%)$ & 28 \\
Women & & $20 \%$ & 29 \\
40 & $0.1 \%$ & $21 \%(29 \%)$ & 27 \\
$50(55)$ & $0.1 \%(1.0 \%)$ & $21 \%(29)$ \\
$60(65)$ & $0.7 \%(1.2 \%)$ & $21 \%(29 \%)$ & 27 \\
$70(75)$ & $2.2 \%(6.2 \%)$ & $20 \%(28 \%)$ & 25 \\
\hline
\end{tabular}

*Hypertension: systolic blood pressure $>160 \mathrm{~mm} \mathrm{Hg}$, or diastolic blood pressure $>100 \mathrm{~mm} \mathrm{Hg}$, or on antihypertensive medication. Rotterdam study data between parentheses.

Table 11 Treatment of hypertension and occurrence of heart failure: meta-analyses ${ }^{\text {w65 }}$ w66

\begin{tabular}{ll}
\hline & $\begin{array}{l}\text { RR for developing heart } \\
\text { failure } \\
(95 \% \mathrm{CI})\end{array}$ \\
\hline$\beta$-blocker vs placebo & $0.58(0.40$ to 0.84$)$ \\
Diuretic (low dose) vs placebo & $0.58(0.44$ to 0.76$)$ \\
Diuretic (high dose) vs placebo & $0.17(0.07$ to 0.41$)$ \\
ACE inhibitor vs placebo & $0.84(0.68$ to 1.04$)$ \\
Calcium channel blocker vs placebo & $0.72(0.48$ to 1.07$)$ \\
\hline
\end{tabular}

$A C E$, angiotensin converting enzyme; $\mathrm{Cl}$, confidence interval; $\mathrm{RR}$, relative risk.

developed heart failure and $273(12.9 \%)$ were admitted for heart failure.

In patients with asymptomatic left ventricular systolic dysfunction following myocardial infarction, both ACE inhibitors and $\beta$-blockers reduce the risk of heart failure. ${ }^{\mathrm{w} 67}$ w68 The SOLVD prevention study demonstrated that ACE inhibition in patients with asymptomatic left ventricular systolic dysfunction (but no recent myocardial infarction) reduces the occurrence of heart failure. The effect on mortality was not significant, leading the authors to conclude "...there may be only a small difference in mortality between asymptomatic patients treated preventively and those treated with careful follow-up and initiation of therapy if heart failure develops".w14

The introduction of neurohormonal markers (especially Btype natriuretic peptide) and portable ultrasound machines (offering the possibility of simultaneous screening for left ventricular systolic dysfunction and abdominal aortic aneurysms) appears to make screening more feasible. It is conceivable that screening of persons at increased risk of having asymptomatic left ventricular systolic dysfunction may be costeffective. As long as prospective studies to determine if screening for asymptomatic left ventricular systolic dysfunction improves prognosis are lacking, screening cannot be recommended, however. ${ }^{\text {w69 }}$

Additional references appear on the Heart website- http:// heart.bmj.com/supplemental 


\section{INTERACTIVE MULTIPLE CHOICE QUESTIONS}

This Education in Heart article has an accompanying series of six EBAC accredited multiple choice questions (MCQs).

To access the questions, click on BMJ Learning: Take this module on BMJ Learning from the content box at the top right and bottom left of the online article. For more information please go to: http://heart.bmj.com/misc/education.dtl Please note: The MCQs are hosted on BMJ Learning-the best available learning website for medical professionals from the BMJ Group.

If prompted, subscribers must sign into Heart with their journal's username and password. All users must also complete a one-time registration on BMJ Learning and subsequently log in (with a BMJ Learning username and password) on every visit.

\section{Authors' affiliations}

Arend Mosterd*, Department of Cardiology, Meander Medical Centre, Amersfoort, The Netherlands

Arno W Hoes, Julius Centre for Health Sciences and Primary Care,

University Medical Centre, Utrecht, The Netherlands

*Also Julius Centre for Health Sciences and Primary Care, and Department of Cardiology, Heart Lung Institute, University Medical Centre, Utrecht, The Netherlands

In compliance with EBAC/EACCME guidelines, all authors participating in Education in Heart have disclosed potential conflicts of interest that might cause a bias in the article

\section{REFERENCES}

1 Cowie MR, Mosterd A, Wood DA, et al. The epidemiology of heart failure. Eur Heart $J$ 1997; 18:208-25.

- Extensive review of the epidemiology of heart failure.

2 McMurray JJ, Pfeffer MA. Heart failure. Lancet 2005;365:1877-89.

- Review of the current state of the art in heart failure.

3 Remes J, Miettinen H, Reunanen A, et al. Validity of clinical diagnosis of heart failure in primary health care. Eur Heart $J 1991 ; 12: 315-21$.

- First paper to address the limited value of symptoms and signs in diagnosing heart failure.

4 Cowie MR, Struthers AD, Wood DA, et al. Value of natriuretic peptides in assessment of patients with possible new heart failure in primary care. Lancet 1997;350:1349-53.

- First paper to address the value of natriuretic peptides in the diagnosis of heart failure in a population based study.

5 Hogg K, Swedberg K, McMurray J. Heart failure with preserved left ventricular systolic function; epidemiology, clinical characteristics, and prognosis. J Am Coll Cardiol 2004;43:317-27.

- Excellent review of the epidemiology of heart failure with preserved left ventricular systolic function ("diastolic heart failure").

6 Owan TE, Redfield MM. Epidemiology of diastolic heart failure. Prog Cardiovasc Dis 2005;47:320-32.

- Excellent review of the epidemiology of heart failure with preserved left ventricular systolic function ("diastolic heart failure").

7 McDonagh TA, Morrison CE, Lawrence A, et al. Symptomatic and asymptomatic left-ventricular systolic dysfunction in an urban population. Lancet 1997;350:829-33.

- First paper to document the importance of asymptomatic left ventricular systolic dysfunction in the population.

8 Redfield MM, Jacobsen SJ, Burnett JC Jr, et al. Burden of systolic and diastolic ventricular dysfunction in the community: appreciating the scope of the heart failure epidemic. JAMA 2003;289:194-202.

- Very elegant population based study of the prevalence of both left ventricular systolic and diastolic dysfunction in relation to symptoms and signs.

9 Wang TJ, Evans JC, Benjamin EJ, et al. Natural history of asymptomatic left ventricular systolic dysfunction in the community. Circulation 2003;108:977-82.

- First paper to describe the natural history of asymptomatic left ventricular systolic dysfunction.
10 Rutten FH, Grobbee DE, Hoes AW. Differences between general practitioners and cardiologists in diagnosis and management of heart failure: a survey in every-day practice. Eur J Heart Fail 2003;5:337-44.

- Study highlighting the differences in patients with heart failure managed by cardiologists and general practitioners.

11 Mendez GF, Cowie MR. The epidemiological features of heart failure in developing countries: a review of the literature. Int J Cardiol 2001;80:213-9.

- Review of the epidemiology of heart failure in developing countries.

12 Maclntyre K, Capewell S, Stewart S, et al. Evidence of improving prognosis in heart failure: trends in case fatality in 66547 patients hospitalized between 1986 and 1995. Circulation 2000;102:1126-31.

- First paper to report a decline in age adjusted hospitalisation rates for heart failure.

13 Owan TE, Hodge DO, Herges RM, et al. Trends in prevalence and outcome of heart failure with preserved ejection fraction. N Engl J Med 2006;355:251-9.

- First paper to address trends in prevalence and outcomes for heart failure patients with preserved as well as impaired left ventricular systolic function.

14 Bonneux L, Barendregt JJ, Meeter K, et al. Estimating clinical morbidity due to ischemic heart disease and congestive heart failure: the future rise of heart failure. Am J Public Health 1994:84:20-8.

- Seminal paper on the heart failure epidemic.

15 Levy D, Larson MG, Vasan RS, et al. The progression from hypertension to congestive heart failure. JAMA 1996;275:1557-62.

- Paper addressing the importance of hypertension vis a vis coronary artery disease in the occurrence of heart failure.

16 Fox KF, Cowie MR, Wood DA, et al. Coronary artery disease as the cause of incident heart failure in the population. Eur Heart $J$ 2001;22:228-36.

- Study documenting the importance of vigorously evaluating patients with heart failure to establish the cause of heart failure.

17 Kenchaiah S, Narula J, Vasan RS. Risk factors for heart failure. Med Clin North Am 2004:88:1145-72.

- Excellent review of risk factors for the occurrence of heart failure.

18 Hellermann JP, Goraya TY, Jacobsen SJ, et al. Incidence of heart failure after myocardial infarction: is it changing over time? Am J Epidemiol 2003:157:1101-7.

- Excellent paper documenting that improved survival following myocardial infarction does not contribute to the heart failure epidemic.

19 Kenchaiah S, Evans JC, Levy D, et al. Obesity and the risk of heart failure. N Engl J Med 2003;347:305-13.

- First study to document the importance of obesity, adjusted for known risk factors, as a risk factor for heart failure.

20 Kalantar-Zadeh K, Block G, Horwich T, et al. Reverse epidemiology of conventional cardiovascular risk factors in patients with chronic heart failure. J Am Coll Cardiol 2004;43:1439-44.

- Paper addressing the phenomenon of "reverse epidemiology" in patients with heart failure - that is, conventional risk factors, blood pressure, obesity, cholesterol, being "protective" in patients with heart failure.

21 Suskin N, Sheth T, Negassa A, et al. Relationship of current and past smoking to mortality and morbidity in patients with left ventricular dysfunction. J Am Coll Cardiol 2001;37:1677-82.

- Study highlighting the beneficial effect of quitting smoking in patients with heart failure.

22 Rutten FH, Cramer MJ, Grobbee DE, et al. Unrecognized heart failure in elderly patients with stable chronic obstructive pulmonary disease. Eur Heart $J$ 2005;26:1887-94.

- First study to address the under appreciation of heart failure in patients with pulmonary disease.

23 Roger VL, Weston SA, Redfield MM, et al. Trends in heart failure incidence and survival in a community-based population. JAMA 2004;292:344-50.

- Population-based study on trends in incidence and survival of heart failure.

24 Levy WC, Mozaffarian D, Linker DT, et al. The Seattle Heart Failure Model: prediction of survival in heart failure. Circulation 2006;113:1424-33.

- Extensively validated model to predict prognosis in heart failure patients.

25 Lloyd-Jones DM, Larson MG, Leip EP, et al. Lifetime risk for developing congestive heart failure: the Framingham heart study. Circulation 2003;106:3068-72.

- First paper to present lifetime risks of the occurrence of heart failure, including the detrimental effect of hypertension.

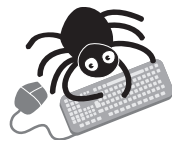

Additional references appear on the Heart websitehttp://heart.bmi.com/supplemental 